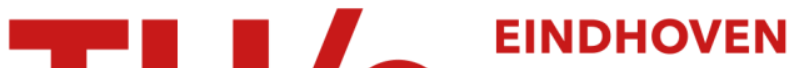 UNIVERSITY OF TECHNOLOGY
}

\section{Structural Transitions in Colloidal Suspensions}

Citation for published version (APA):

Hecht, M., \& Harting, J. D. R. (2007). Structural Transitions in Colloidal Suspensions. In W. E. Nagel, D. Kröner, \& M. Resch (Eds.), High Performance Computing in Science and Engineering '07 (pp. 45-65). Springer. https://doi.org/10.1007/978-3-540-74739-0_4

DOI:

10.1007/978-3-540-74739-0_4

Document status and date:

Published: 01/01/2007

\section{Document Version:}

Publisher's PDF, also known as Version of Record (includes final page, issue and volume numbers)

\section{Please check the document version of this publication:}

- A submitted manuscript is the version of the article upon submission and before peer-review. There can be important differences between the submitted version and the official published version of record. People interested in the research are advised to contact the author for the final version of the publication, or visit the $\mathrm{DOI}$ to the publisher's website.

- The final author version and the galley proof are versions of the publication after peer review.

- The final published version features the final layout of the paper including the volume, issue and page numbers.

Link to publication

\section{General rights}

Copyright and moral rights for the publications made accessible in the public portal are retained by the authors and/or other copyright owners and it is a condition of accessing publications that users recognise and abide by the legal requirements associated with these rights.

- Users may download and print one copy of any publication from the public portal for the purpose of private study or research.

- You may not further distribute the material or use it for any profit-making activity or commercial gain

- You may freely distribute the URL identifying the publication in the public portal.

If the publication is distributed under the terms of Article $25 \mathrm{fa}$ of the Dutch Copyright Act, indicated by the "Taverne" license above, please follow below link for the End User Agreement:

www.tue.nl/taverne

Take down policy

If you believe that this document breaches copyright please contact us at:

openaccess@tue.nl

providing details and we will investigate your claim. 


\title{
Structural Transitions in Colloidal Suspensions
}

\author{
Martin Hecht and Jens Harting \\ Institut für Computerphysik, Pfaffenwaldring 27, 70569 Stuttgart, Germany
}

\begin{abstract}
Summary. In suspensions of colloidal particles different types of interactions are in a subtle interplay. In this report we are interested in sub-micro meter sized $\mathrm{Al}_{2} \mathrm{O}_{3}$ particles which are suspended in water. Their interactions can be adjusted by tuning the $p \mathrm{H}$-value and the salt concentration. In this manner different microscopic structures can be obtained. Industrial processes for the production of ceramics can be optimized by taking advantage of specific changes of the microscopic structure. To investigate the influences of the $p \mathrm{H}$-value and the salt concentration on the microscopic structure and the properties of the suspension, we have developed a coupled Stochastic Rotation Dynamics (SRD) and Molecular Dynamics (MD) simulation code. The code has been parallelized using MPI. We utilize the pair correlation function and the structure factor to analyze the structure of the suspension. The results are summarized in a stability diagram. For selected conditions we study the process of cluster formation in large scale simulations of dilute suspensions.
\end{abstract}

Key words: Stochastic Rotation Dynamics; Molecular Dynamics; colloids; clustering

\section{Introduction}

Colloid science is a very fascinating research field, gaining more and more importance in the last years. It closely connects physics, chemistry, material science, biology, and several branches of engineering technology. According to its key role in modern science a considerable amount of research has been performed to describe colloidal suspensions from a theoretical point of view and by simulations $[16,28,29,41,47,49]$ as well as to understand the particleparticle interactions $[3,11,12,15,51,52]$, the phase behavior $[10,23,32,50]$, the relevant processes on the microscale and their influence on macroscopic parameters [13, 40, 54]. Colloidal suspensions are in fact complicated systems, since depending on the particle sizes, materials, and concentrations, different interactions are of relevance and often several of them are in a subtle interplay: electrostatic repulsion, depletion forces, van der Waals attraction, 
hydrodynamic interaction, Brownian motion, and gravity are the most important influences. The properties of the suspension strongly depend on the balance of the microscopic forces between the particles. Especially for industrial processes, where one needs to optimize certain material properties a detailed understanding of the relevant influences is needed. The stability of different microstructures and especially the clustering process are key properties which are of interest.

In our work we investigate these properties, focusing on $\mathrm{Al}_{2} \mathrm{O}_{3}$ particles suspended in water. This is a widely used material in ceramics [37]. We have developed a simulation code for a Brownian suspension [20] and have adjusted the simulation parameters so that the simulation corresponds quantitatively to a real suspension such that experimental data can be compared directly. The diffusion coefficient, sedimentation velocity [20], and the viscosity of the suspension can be reproduced [17]. We also have tested the influence of polydispersity and found that its influence on the results is small. It is much more important to choose the correct mean size of the particles [17]. For $\mathrm{Al}_{2} \mathrm{O}_{3}$ suspensions attractive van der Waals forces are important for the behavior of this material. Electrostatic repulsion of the charged particles counteracts the attraction and can prevent clustering depending on the particle surface charge. In [17] we have presented how one can relate parameters of DLVO potentials $[11,52]$ with experimental conditions. In the experiment one can control the $p \mathrm{H}$-value and the salt concentration. The latter can be expressed by the ionic strength $I$, which is an effective concentration of all ions present in the solution. Both, the $p \mathrm{H}$-value and the ionic strength, influence the charge of the colloidal particles. We have shown that for not too strongly attractive forces one can obtain reasonable quantitative agreement with experimental results.

Three regimes can be identified and plotted in a stability diagram [17], which we want to investigate here in more detail: A clustered regime, in which particles aggregate to clusters, a fluid-like and stable stable suspension and a repulsive region, for which the microstructure is similar to the ones known from glassy systems. From our previous work we know that our model works well, even quantitatively, in the suspended regime of the stability diagram and close to the borders between the different microstructures. Here we extend our investigations to different $p \mathrm{H}$-values, deeper in the clustered regime, and to the repulsive structure. We expect to gain insight to the microscopic structure on a qualitative level.

On these grounds we have explored the stability diagram of $\mathrm{Al}_{2} \mathrm{O}_{3}$ suspensions. The particles are uncharged close to the so called "isoelectric point" at $p \mathrm{H}=8.7$. There, for all ionic strengths the particles form clusters. For lower $p \mathrm{H}$-values particles can be stabilized in solution by the electrostatic repulsion due to the charge the particles carry in this case. For low $p \mathrm{H}$-values, low salt concentrations, and high volume fractions a repulsive structure can be found.

In the following section we shortly describe our simulation method. After that we discuss the properties which can be found in our suspensions and how 
different regimes of the stability diagram are distinguished. In the section thereafter we describe our simulation setup. Then, we present our results and discuss the criteria we apply to characterize the microstructures. We utilize the pair correlation function and the structure factor to characterize the clustering behavior. Both of them in principle contain the same information, but we concentrate on certain peaks in either of them. Each peak in the correlation function and in the structure factor corresponds to a certain length scale and we chose either the correlation function or the structure factor, depending on which of the two quantities is more suitable under numerical criterions to observe on a given length scale. To characterize the repulsive region we evaluate the mean squared displacement (MSD), which shows a plateau, if the particle motion consists of different processes acting on well separated time scales. Finally, the results are summarized in a stability diagram for our $\mathrm{Al}_{2} \mathrm{O}_{3}$-suspension. It shows the behavior of the suspension in an intuitive way and helps to design industrial processes using this material. After that, we turn to dilute suspensions of only $5 \%$ volume fraction and study cluster growth at low shear rates in these suspensions. Finally, we shortly summarize our results. The results which we present in this report have been accepted for publication in $[18,19]$.

\section{Simulation Method}

Our simulation method is described in detail in $[17,20]$ and consists of two parts: a Molecular Dynamics (MD) code, which treats the colloidal particles, and a Stochastic Rotation Dynamics (SRD) simulation for the fluid solvent. In the MD part we include effective electrostatic interactions and van der Waals attraction, known as DLVO potentials [11, 52], a lubrication force and Hertzian contact forces. DLVO potentials are composed of two terms, the first one being an exponentially screened Coulomb potential due to the surface charge of the suspended particles

$$
V_{\text {Coul }}=\pi \varepsilon_{r} \varepsilon_{0}\left[\frac{2+\kappa d}{1+\kappa d} \cdot \frac{4 k_{\mathrm{B}} T}{z e} \tanh \left(\frac{z e \zeta}{4 k_{\mathrm{B}} T}\right)\right]^{2} \times \frac{d^{2}}{r} \exp (-\kappa[r-d]),
$$

where $d$ denotes the particle diameter, $r$ the distance between the particle centers, $e$ the elementary charge, $T$ the temperature, $k_{\mathrm{B}}$ the Boltzmann constant, and $z$ is the valency of the ions of added salt. $\varepsilon_{0}$ is the permittivity of the vacuum, $\varepsilon_{r}=81$ the relative dielectric constant of the solvent, $\kappa$ the inverse Debye length defined by $\kappa^{2}=8 \pi \ell_{B} I$, with ionic strength $I$ and Bjerrum length $\ell_{B}=7$. The effective surface potential $\zeta$ can be related to the $p H$-value of the solvent with a $2 p K$ charge regulation model [17]. The Coulomb term competes with the attractive van der Waals interaction $\left(A_{\mathrm{H}}=4.76 \cdot 10^{-20} \mathrm{~J}\right.$ is the Hamaker constant) [21]

$$
V_{\mathrm{VdW}}=-\frac{A_{\mathrm{H}}}{12}\left[\frac{d^{2}}{r^{2}-d^{2}}+\frac{d^{2}}{r^{2}}+2 \ln \left(\frac{r^{2}-d^{2}}{r^{2}}\right)\right] .
$$


The attractive contribution $V_{\mathrm{VdW}}$ is responsible for the cluster formation we observe. However, depending on the $p \mathrm{H}$-value and the ionic strength, it may be overcompensated by the electrostatic repulsion. When particles get in contact, the potential has a minimum. However, (2) diverges due to the limitations of DLVO theory. We cut off the DLVO potentials and model the minimum by a parabola as described in [20]. The particle contacts are modeled as Hertzian contacts and for non-touching particles. Below the resolution of the SRD algorithm short range hydrodynamics is corrected by a lubrication force, which we apply within the MD framework, as we have explained in [17, 20]. For the integration of translational motion of the colloidal particles we utilize a velocity Verlet algorithm [5].

For the simulation of a fluid solvent, many different simulation methods have been proposed: Stokesian Dynamics (SD) [6, 7, 41], Accelerated Stokesian Dynamics (ASD) [45, 46], pair drag simulations [47], Brownian Dynamics (BD) [21, 22], Lattice Boltzmann (LB) [27, 28, 29, 30], and Stochastic Rotation Dynamics (SRD) [20, 26, 38]. These mesoscopic fluid simulation methods have in common that they make certain approximations to reduce the computational effort. Some of them include thermal noise intrinsically, or it can be included consistently. They scale differently with the number of embedded particles and the complexity of the algorithm differs largely.

We apply the Stochastic Rotation Dynamics method (SRD) introduced by Malevanets and Kapral [33, 34]. It intrinsically contains fluctuations, is easy to implement, and has been shown to be well suitable for simulations of colloidal and polymer suspensions $[4,17,20,26,38,42,53]$ and recently for star-polymers in shear flow [44]. The method is also known as "Real-coded Lattice Gas" [26] or as "multi-particle-collision dynamics" (MPCD) [43]. It is based on so-called fluid particles with continuous positions and velocities. A streaming step and an interaction step are performed alternately. In the streaming step, each particle $i$ is moved according to

$$
\mathbf{r}_{i}(t+\tau)=\mathbf{r}_{i}(t)+\tau \mathbf{v}_{i}(t)
$$

where $\mathbf{r}_{i}(t)$ denotes the position of the particle $i$ at time $t$ and $\tau$ is the time step. In the interaction step the fluid particles are sorted into cubic cells of a regular lattice and only the particles within the same cell interact among each other according to an artificial interaction. The interaction step is designed to exchange momentum among the particles, but at the same time to conserve total energy and total momentum within each cell, and to be very simple, i.e., computationally cheap: each cell $j$ is treated independently. First, the mean velocity $\mathbf{u}_{j}\left(t^{\prime}\right)=\frac{1}{N_{j}\left(t^{\prime}\right)} \sum_{i=1}^{N_{j}\left(t^{\prime}\right)} \mathbf{v}_{i}(t)$ is calculated. $N_{j}\left(t^{\prime}\right)$ is the number of fluid particles contained in cell $j$ at time $t^{\prime}=t+\tau$. Then, the velocities of each fluid particle in cell $j$ are rotated according to

$$
\mathbf{v}_{i}(t+\tau)=\mathbf{u}_{j}\left(t^{\prime}\right)+\boldsymbol{\Omega}_{j}\left(t^{\prime}\right) \cdot\left[\mathbf{v}_{i}(t)-\mathbf{u}_{j}\left(t^{\prime}\right)\right]
$$

$\boldsymbol{\Omega}_{j}\left(t^{\prime}\right)$ is a rotation matrix, which is independently chosen at random for each time step and each cell. We use rotations about one of the coordinate axes 
by an angle $\pm \alpha$, with $\alpha$ fixed. The coordinate axis as well as the sign of the rotation are chosen at random, resulting in 6 possible rotation matrices. To remove anomalies introduced by the regular grid, one can either choose a mean free path of the order of the cell size or shift the whole grid by a random vector once per SRD time step as proposed by Ihle and Kroll [24, 25].

Three different methods to couple the SRD and the MD simulation have been introduced in the literature. Inoue et al. proposed a way to implement no slip boundary conditions on the particle surface [26]. Padding and Louis very recently came up with full slip boundaries, where the fluid particles interact via Lennard-Jones potentials with the colloidal particles [39]. Falck et al. [14] have developed a "more coarse grained" method which we use for our simulations and which we descibe shortly in the following.

To couple the colloidal particles to the fluid, the colloidal particles are sorted into the SRD cells and their velocities are included in the rotation step. One has to use the mass of each particle -colloidal or fluid particle -as a weight factor when calculating the mean velocity

$$
\begin{aligned}
\mathbf{u}_{j}\left(t^{\prime}\right) & =\frac{1}{M_{j}\left(t^{\prime}\right)} \sum_{i=1}^{N_{j}\left(t^{\prime}\right)} \mathbf{v}_{i}(t) m_{i}, \\
\text { with } \quad M_{j}\left(t^{\prime}\right) & =\sum_{i=1}^{N_{j}\left(t^{\prime}\right)} m_{i},
\end{aligned}
$$

where we sum over all colloidal and fluid particles in the cell, so that $N_{j}\left(t^{\prime}\right)$ is the total number of both particles, fluid plus colloidal ones. $m_{k}$ is the mass of the particle with index $i$ and $M_{j}\left(t^{\prime}\right)$ gives the total mass contained in cell $j$ at time $t^{\prime}=t+\tau$. To some of our simulations we apply shear. This is realized by explicitly setting the mean velocity $\mathbf{u}_{j}$ to the shear velocity in the cells close to the border of the system. Both, colloidal and fluid particles, are involved in this additional step. A thermostat is applied to remove the energy introduced to the system by the shear force. We have described the simulation method in more detail in $[17,20]$.

A single simulation run as presented in these papers took between one and seven days on a $3 \mathrm{GHz}$ Pentium CPU. However, for strongly clustering systems we easily end up with only a single cluster inside the simulation volume. In order to be able to gather statistics on cluster growth and formation, as well as to minimize finite size effects, we parallelized our code. While MD codes have been parallelized by many groups, only few parallel implementations of a coupled MD and SRD program exist. This is in contrast to the number of parallel implementations of other mesoscopic simulation methods like for example the lattice Boltzmann method. A possible explanation is that $\mathrm{SRD}$ is a more recent and so far not as widely used algorithm causing the parallelization to be a more challenging task.

We utilize the Message Pasing Interface (MPI) to create a $\mathrm{C}++$ code based on domain decomposition for both involved simulation methods. In 
the MD code the position of neighbouring particles is needed to compute the interactions. Since the intractions have a limited range, and a linked cell algorithm is already used in the serial code, we apply linked cells here as well. Particle positions at the border of the domain of each processor are communicated to the neighbouring processors for the calculation of the forces. Then, the propagation step is performed and particle positions are updated, whereby the particles crossing a domain boundary are transferred from one processor to the other one.

Since (in principle), fluid particles can travel arbitrary large distances in one time step, one either has to limit the distance they can move, or one needs all-to-all communication between the processors. Even though the mean free path in our systems is small enough to limit communication to nearest neighbours only, the current version of our code tries to be as general as possible and allows fluid particles to move to any possible position in the total simulation volume within a single timestep. First, we determine locally which fluid particles have to be sent to which destination CPU and collect all particles to be sent to the same destination into a single MPI message. If no particles are to be sent, a zero dummy message is transmitted. On the receiving side, MPI_Probe with the MPI_ANY_SOURCE option is utilized to determine the sender's rank and the number of particles to be accomodated. Now, MPI_Recv can be used to actually receive the message. All processors send and receive in arbitrary order, thus waiting times are kept at a minimum allowing a very efficient communication. The standard MPI all-to-all communication procedure should be less efficient since the size of every message would be given by the size of the largest message. However, we still do find a substantial communication overhead from our benchmark tests of the scalability of the code. Due to this overhead, we are currently limited to 32 CPUs. In order to achieve Gallilean invariance, a random shift of the SRD lattice is performed for every rotation step $[24,25]$. Since the domains managed by each CPU do not move, this would include the borders between the processors to cross SRD cells, which is undesirable. Therefore, we keep the position of the lattice fixed and shift the fluid particle positions before sorting them into the cells instead. After the rotation step they are shifted back.

\section{Background}

We examine the microstructures obtained in our simulations for different conditions. We vary the $p \mathrm{H}$-value and the ionic strength $I$. The shear rate $\dot{\gamma}$ as an external influence is varied as well. We classify the microstructures in three categories: suspended, clustered, and repulsive. In the suspended case, the particles can move freely in the fluid and do not form stable clusters. In the clustered regime the particles form clusters due to attractive van der Waals forces. These clusters can be teared apart if shear is applied. In some of our simulations the clusters are very weakly connected and at small shear rates 
they are not only broken up into smaller pieces, but they dissolve to freely moving individual particles. In this case, we assign the microstructure to the suspended region, although in complete absence of the shear flow clusters are formed. At the borders between the different regimes in fact no sharp transitions can be observed. The DLVO forces rather steadily increase and compete with the hydrodynamic interactions. Accordingly, in experiments one cannot observe a sudden solidification, but a steadily increasing viscosity when leaving the suspended regime [17].

Similarly as for attractive forces, repulsive interactions can restrict the mobility of the particles. If this happens, the mean squared displacement of the particles shows a pronounced plateau, as it can be found in glassy systems. However, we speak of a "repulsive structure", because the change of the viscosity is not as strong as in glasses, where it often changes by many oders of magnitude, when the glass transition is approached. In addition, to claim a system shows a glassy behavior would require to investigate the temperature dependence of a typical time (e.g. particle diffusion time) and to show its divergence as the glass temperature is approached. This is difficult to do in the framework of our simulation model [20] and therefore we prefer to speak about a "repulsive structure" which might be identified as a colloidal glass in future work.

Here we would like to emphasize the analysis of the microstructure for different conditions. Our aim is to reproduce a so-called stability diagram by simulations. The stability diagram depicts the respective microstructure depending on the $p \mathrm{H}$-value and the ionic strength $I$. We apply different numerical tools to analyze the microstructure in our simulations and finally arrive at a stability diagram shown in Fig. 7, which summarizes the results which we present in the following sections.

\section{Simulation Setup}

In this study the colloidal particles are represented by three dimensional spheres of $d=0.37 \mu \mathrm{m}$ in diameter. This is the mean diameter of the particles used in the experiments to which we refer in [17]. We have simulated a small volume, $24 d=8.88 \mu \mathrm{m}$ long in $x$-direction, which is the shear direction, and $12 d=4.44 \mu \mathrm{m}$ long in $y$ - and $z$-direction. We have varied the volume fraction between $\Phi=10 \%$ (660 particles) and $\Phi=40 \%$ (2640 particles). Most of the simulations were performed at $\Phi=35 \%$ (2310 particles). To study low volume fractions $\Phi=5 \%$ we have enlarged the simulation volume to $24 d=8.88 \mu \mathrm{m}$ in each direction and we have further scaled up the system in each dimesion by a factor of 2 or 4 , resulting in a cube of $48 d=17.76 \mu \mathrm{m}$. For selected $p \mathrm{H}$-values and ionic strengths we have studied the cluster growth of dilute suspensions at low shear rates $(\dot{\gamma}=20 /$ s $)$.

We use periodic boundaries in $x$ - and $y$-direction and closed boundaries in $z$-direction [17]. Shear is applied in $x$-direction by moving small zones of 
particles and fluid close to the wall with a given shear velocity. The $x y$-plane is our shear plane. For simulations without shear, to achieve the best comparability, we use the same boundary conditions and just set the shear rate to $\dot{\gamma}=0$. In addition we have performed simulations with two different shear rates: with $\dot{\gamma}=100 /$ s and with $\dot{\gamma}=500 /$ s.

\section{Results and Discussion}

First, we focus on simulations without shear, where one can predict intuitively, what should happen. Qualitatively the results are similar to our earlier work [20], but the quantitative relation between the $p \mathrm{H}$-value and the potentials is new. The relation was presented in [17], but here we apply it to different cases and we focus more on the characterization of the microstructure. However, given the particle particle interaction potentials, the microstructure in equilibrium can be predicted easily, at least on a qualitative level. But, the matter changes and gets more sophisticated, when shear is applied and an interplay between shear flow and particle particle interactions becomes responsible for the resulting microstructure. At the end of this section we move on to dilute suspensions and study the growth of clusters at low shear rates.

\subsection{Correlation Function}

For constant ionic strength $I=3 \mathrm{mmol} / 1$ the local microstructure can be examined using the correlation function. Depending on the $p \mathrm{H}$-value the behavior of the system changes from a repulsive structure around $p \mathrm{H}=4$ to a stable suspension around $p \mathrm{H}=6$ towards a clustered region if the $p \mathrm{H}$-value is further increased, until the isoelectric point is reached at $p \mathrm{H}=8.7$. There clustering occurs in any case, independent on the ionic strength. This can be seen in the structure of the correlation function

$$
g(r)=\frac{V}{N^{2}}\left\langle\sum_{i} \sum_{j \neq i} \delta\left(r-r_{i j}\right)\right\rangle,
$$

(see [5] p. 55), where $V$ is the volume, $N$ the number of particles and $r_{i j}$ the distance of two particles $i$ and $j$.

At $p \mathrm{H}=4$ electrostatic repulsion prevents clustering: Particles are suspended, and there is no fixed long range ordering in the system. The correlation function (Fig. 1) shows a maximum at a typical nearest neighbor distance slightly above $\frac{r}{d}=1$ with $d$ denoting the particle diameter, then in the layer of next neighbors small correlations can be found (at $\frac{r}{d}=2$ ). For larger distances the correlation function is rather constant.

When the $p \mathrm{H}$-value is increased, the surface charge is lower, which at first causes the particles to approach each other more closely. The maximum of the correlation function is shifted to smaller distances (see Fig. 1, note that the 
curves are shifted vertically in the plot by a factor of 3 for better visibility.). Then, van der Waals attraction becomes more important and clustering begins. One can see this in the correlation function where a sharp structure at particle distances between 1.5 and 2 particle diameters occurs. There is a nearest neighbor peak, and more complicated structures at larger distances, which we have assigned to typical particle configurations for small distances [20]. In a solid like cluster the position of the next neighbor is fixed more sharply than in the suspension, consequently the nearest neighbor peak becomes sharper, and its height is increased. Close to the isoelectric point $(p \mathrm{H}=8.7)$ the barrier between primary and secondary minimum disappears. The particles, once clustered, cannot rearrange anymore, and therefore the correlations to the next neighbors become less sharp again (compare the cases of $p \mathrm{H}=8.7$ and $p \mathrm{H}=7.7$ in Fig. 1 at the positions denoted by the arrows).

Instead of varying the $p \mathrm{H}$-value, one can also vary the ionic strength to achieve similar effects. Increasing the ionic strength, experimentally speaking "adding salt" decreases the screening length $1 / \kappa$ and therefore the attractive forces become more important: the particles start to form clusters.

The effects described up to here can be observed with or without shear qualitatively in an analogous manner. If the suspension is sheared clustering occurs at higher $p \mathrm{H}$-values and the peaks found in the correlation function are slightly broadened, because the relative particle positions are less fixed. But a new feature appears, if a stable suspension of not too high volume fraction is sheared. Induced by the shear particles arrange themselves in layers. Regular nearest neighbor distances in the shear plane cause the correlation function to

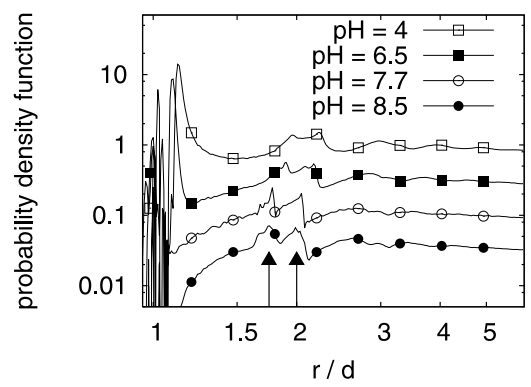

Fig. 1. Dependence of the particle correlation function on the $\mathrm{pH}$ value, $I=3 \mathrm{mmol}$, $\dot{\gamma}=0 / \mathrm{s} \Phi=35 \%$. The plots for four different $p \mathrm{H}$-values are shifted against each other for better visibility by a factor of 3 . For $p \mathrm{H}=4$ the particles are not clustered. Hence the structure at $\frac{r}{d}=2$ is less sharp than in the other three curves of the plot and the nearest neighbor peak (at $\frac{r}{d}=1$ ) is broad. For $p \mathrm{H}=6.5$ slight clustering starts, the structures become sharper. For $p \mathrm{H}=7.7$ strong cluster formation is reflected in very sharp structures. For $p \mathrm{H}=8.5$ electrostatic repulsion nearly disappears so that no barrier between primary and secondary minimum exists anymore. The particles cannot rearrange anymore, and therefore the structures labeled by the arrows become smoothened compared to the case of $p \mathrm{H}=7.7$ (source: [19]) 


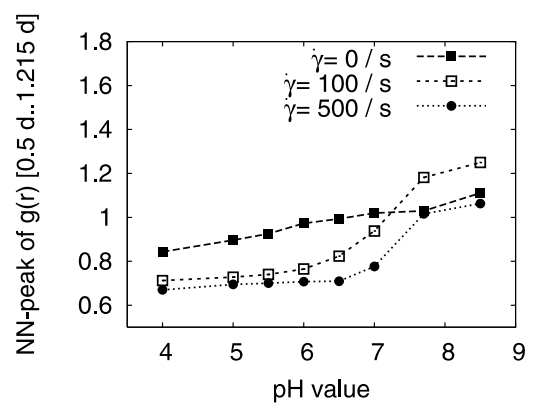

Fig. 2. Nearest neighbor peak (primary and secondary minimum of the potential) of the correlation function $I=3 \mathrm{mmol} / \mathrm{l}, \Phi=35 \%$ : For low $p \mathrm{H}$-values clustering is prevented by the electrostatic repulsion. For high $p \mathrm{H}$-values the particles form clusters, which is reflected by an increased nearest neighbor peak. First, shear prevents clustering, then depending on the shear rate, cluster formation takes place. Low shear rates even support cluster formation at high $p \mathrm{H}$-values (source: [19])

become more structured even for large distances. The long range structure of the pair correlation function appears after a transient time the particles need to arrange themselves in the layered structure. Shear induced layer formation has been found in both, experiments $[1,2]$ and simulations $[8,9,36]$

We have integrated over the nearest neighbor peaks, both, the peaks of the primary and the secondary minimum, and plotted the integral versus $p \mathrm{H}$-value in Fig. 2. We have chosen $I=3 \mathrm{mmol} / \mathrm{l}$ and $\Phi=35 \%$ and three different shear rates: $\dot{\gamma}=0,100$ and $500 / \mathrm{s}$. We have integrated the correlation function for $r<1.215 d$, where for all $p \mathrm{H}$-values the potential in the secondary minimum has a value of $-\frac{1}{2} k_{\mathrm{B}} T$. In other words, we have captured the primary and the secondary minimum of the potential for this plot. For low $p \mathrm{H}$-values clustering (in the secondary minimum) is only possible for low shear rates. For high shear rates, the hydrodynamic forces do not allow the formation of stable clusters. For rising $p \mathrm{H}$-values the clustering increases, first for the un-sheared suspension, at higher $p \mathrm{H}$-values for low shear rates $(\dot{\gamma}=100 / \mathrm{s})$ and finally for high shear rates $(\dot{\gamma}=500 / \mathrm{s})$. Remarkably, for $p \mathrm{H}>7.5$ the curve for $\dot{\gamma}=100 /$ s shows stronger cluster formation than the other ones. Particles are brought together by the shear flow, so that compared to the case of no shear, the clustering process is supported here. On the other hand, the shear stress may not be too strong, because otherwise the clustering process is limited by the shear flow again (for $\dot{\gamma}=500 /$ s the clustering is less pronounced than for $\dot{\gamma}=100 / \mathrm{s})$.

\subsection{Structure Factor}

The pair correlation function can be used to characterize the local order of the microstructure on the length scale of the particle size. However, to do the characterization on the length scale of the system size, we use the structure 
factor defined by

$$
S(\mathbf{k})=\frac{1}{N} \sum_{l, m=1}^{N} \exp \left(i \mathbf{k} \cdot \mathbf{r}_{l m}\right)
$$

where $N$ is the number of particles, and $\mathbf{r}_{l m}$ is the vector from particle $l$ to particle $m$. $i$ denotes the imaginary unit here. The structure factor is related to the pair correlation function in real space by a three dimensional Fourier transform. In principle the structure factor contains the same information as the pair correlation function. However, due to numerical reasons and our implementation of shear boundary conditions it is easier to observe the longrange structure in the structure factor than in the pair correlation function.

In Fig. 3 we have plotted several typical structure factors of our simulations. For these plots the $p \mathrm{H}$-value is fixed to $p \mathrm{H}=6$. The cases a) and $\mathrm{b}$ ) are sheared with $\dot{\gamma}=500 / \mathrm{s}$ at an ionic strength of $I=0.3 \mathrm{mmol} / \mathrm{l}$. In case a) the volume fraction $\Phi=20 \%$ is relatively low. Therefore the particles can arrange themselves in layers parallel to the shear plane, which move relatively independently in the shear flow. They have a certain distance fixed in space and time. This can be seen in a sharp peak at a dimensionless $\mathrm{k}$-vector of $k=5.2$, which corresponds to a distance of 1.2 particle diameters. In fact, this is exactly the distance between two neighboring layers, as one can easily verify by counting the layers in a snapshot of the system (Fig. 4a)). The particles in the layers do not have a fixed distance and therefore no 2nd-order-peak can be observed.

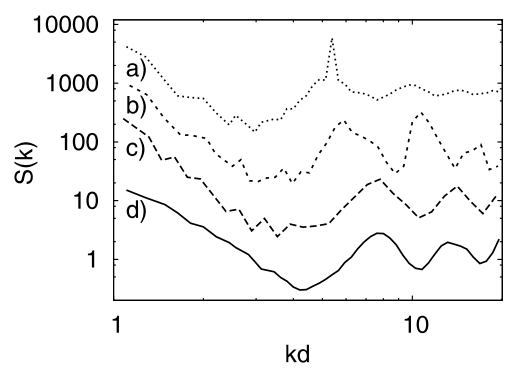

Fig. 3. Structure factor for some selected examples, with $p \mathrm{H}=6$ fixed for all plots: $\dot{\gamma}=500 / \mathrm{s}, I=0.3 \mathrm{mmol} / \mathrm{l}:$ a) $\Phi=20 \%$ and $b) \Phi=35 \%, \dot{\gamma}=0, I=25 \mathrm{mmol} / \mathrm{l}$ : c) $\Phi=40 \%$ and $d$ ) $\Phi=10 \%$. The curves are shifted vertically for better visibility. In case $a$ ) ten layers can be identified in the system, resulting in the strong peak close to 5. But, since the particles in the layers can still move freely, there is no 2nd-order-peak. In case $b$ ) layers are formed, but particles are moving from one layer to the other, disturbing the flow. As a result the nearest neighbor peak is much broader. Due to the structure in the layers, a 2nd-order-peak appears. In case $c$ ) the interaction is strongly attractive, hence the particles approach each other and the nearest neighbor peak is shifted to higher $\mathbf{k}$-vectors. In case $d$ ) the volume fraction is much less. The slope of the low-k-peak is much flatter, which depicts that the cluster is fractal (source: [19]) 
For case b) the volume fraction is increased to $\Phi=35 \%$. The particle layers are packed more densely and therefore the interactions between one layer and the neighboring one become relevant. Particles jump from one layer to the other, which disturbs the flow and therefore the distance between the layers is not fixed anymore. The sharp peak on top of the nearest neighbor peak disappears. Instead of that, in each layer a regular hexagonal order appears and therefore the 2nd-order-peak is much more pronounced.

In case c) the ionic strength is increased to $I=25 \mathrm{mmol} / \mathrm{l}$. The interparticle potentials are attractive enough that aggregation takes place. In this simulation we did not apply shear, therefore one finds only one big cluster in the system (compare Fig. 4c)). In the cluster the particles are packed more densely and consistently the nearest neighbor peak in the structure factor is shifted to larger $\mathbf{k}$-vectors. The volume fraction is $\Phi=40 \%$ in this case.

In case $\mathrm{d}$ ) the volume fraction is decreased to $\Phi=10 \%$. The particles still form clusters, but their mobility is not high enough to create one compact cluster. The system has a fractal structure (see Fig. 4d)). This can be seen in the structure factor as well: The slope for low $\mathbf{k}$-vectors is flatter in this case compared to cases a)-c). A flatter slope of the low-k-peak is typical for structure factors of fractal objects. The fractal dimension of the cluster extracted from the slope of the low-k-peak is 2.5. In experiments this relation is often used to determine the fractal dimension of a sample: Lattuada et al. [31] have evaluated the fractal dimension of agglomerates of latex particles from the slope of the structure factor. McCarthy et al. [35] give an introduction to scattering intensities at fractal objects, without mentioning the structure factor, but their arguments refer to the contribution of the structure factor on the scattering intensity. The underlying mechanism which is responsible for these structures is cluster cluster aggregation [48].

In Fig. 5 we show the dependence of the low-k-peak of the structure factor on the $p \mathrm{H}$-value. Here we have integrated over dimensionless $\mathbf{k}$-vectors smaller than 3 which means, we have captured structures larger than twice a particle diameter. A large integral over the low-k-peak is due to a large inhomogeneity in the system. In one part of the system particles are present and in the other part not. In other words, we observe the process of cluster formation on a length scale of the system size. Without shear, particles cluster in the secondary minimum for all $p H$-values. If the system is slightly sheared $(\dot{\gamma}=$ $100 / \mathrm{s}$ ) clustering is suppressed for low $p \mathrm{H}$-values. Starting at $p \mathrm{H}=6$ cluster formation starts and is even supported by the shear flow for $p \mathrm{H}$-values larger than 7.5. For large shear rates $(\dot{\gamma}=500 / \mathrm{s})$ cluster formation is suppressed by the shear flow. By analyzing the low-k-peak of the structure factor one observes on the length scale of the system size. The same behavior of the system can be seen by analyzing the pair correlation function, as we have already shown in Fig. 2. In that case one analyzes the number of nearest neighbors, that means, one observes the length scale of a particle diameter. Nevertheless, both graphs show the same behavior of the system, i.e., we have 


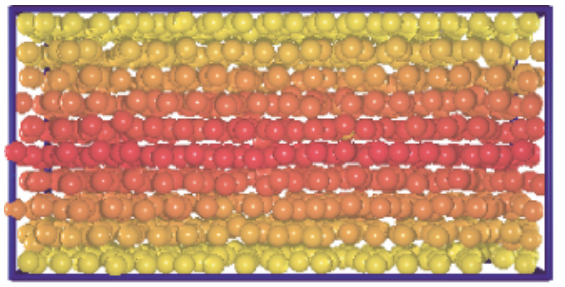

a)

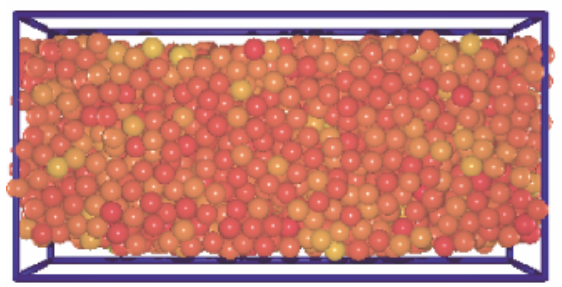

c)

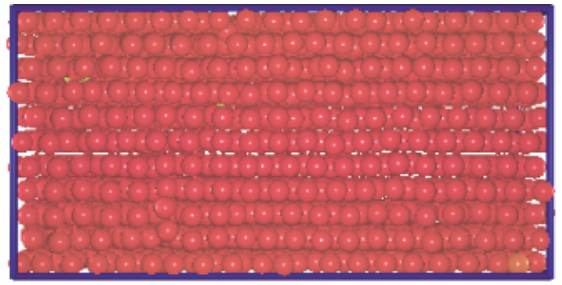

b)

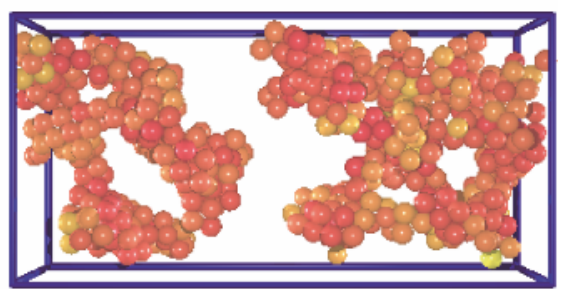

d)

Fig. 4. Snapshots of the systems analyzed in Fig. 3: In case a) one can see the layers resulting in the sharp peak in the structure factor. In case b) the layers are packed closer due to the higher volume fraction. Collisions between particles of neighboring layers happen more frequently. In case c) one big cluster is formed. The particles are packed densely. In case $\mathbf{d}$ ) the fractal nature of the system can be seen directly (source: [19])

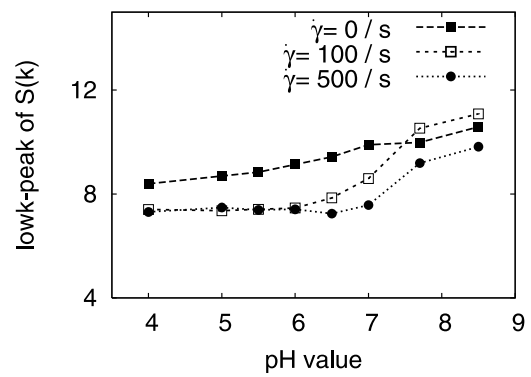

Fig. 5. low-k-peak for different $p \mathrm{H}$-values and different shear rates. The ionic strength $I$ is kept constant at $I=3 \mathrm{mmol} / \mathrm{l}$ and the volume fraction is always $\Phi=35 \%$. For $\dot{\gamma}=0 / \mathrm{s}$ the particles tend to cluster in the secondary minimum of the potential. This clustering can easily be broken up, if shear is applied. If the $p \mathrm{H}$-value is increased, shear cannot prevent cluster formation anymore. At low shear rates $(\dot{\gamma}=100 / \mathrm{s})$ clustering is even enhanced, since the particles are brought closer to each other by the shear flow (source: [19]) 
a consistent picture of the cluster formation process on the length scale of the nearest neighbors and on the length scale of the system size.

Thus we have confirmed that the cluster formation process is not limited to length scales smaller than our system size. This is reflected especially by the transition between $p \mathrm{H}=7-8$ and its shear rate dependence in the plots in Fig. 5 and Fig. 2. There is a strong similarity of the two plots, which are obtained by two evaluation methods referring to two different length scales. This confirms that the plots do not only reflect how clusters are formed on the respective length scale, but that the clustering process is a phenomenon which can be observed on any length scale by applying a suitable method to characterize it.

\subsection{Repulsive Regime}

To characterize the repulsive regime, we evaluate the mean squared displacement for the particles. In Fig. 6 we plot the mean squared displacement for different ionic strengths. The $p \mathrm{H}$-value is kept constant at $p \mathrm{H}=6$ and the volume fraction is $\Phi=35 \%$ for this plot. Three different regimes can be identified. For very short times, the ballistic regime: particles move on short distances without a notable influence by their neighbors. The distances are in the order of some percent of the particle diameter and the times are a few SRD time steps. For larger times the particles interact with their neighbors and therefore their mobility is limited due to collisions with the neighbors. This is reflected in the mean squared displacement by a plateau of reduced slope, which is the more pronounced the more the mobility of the particles is restricted. For even larger time scales collective motion starts, i.e., clusters or

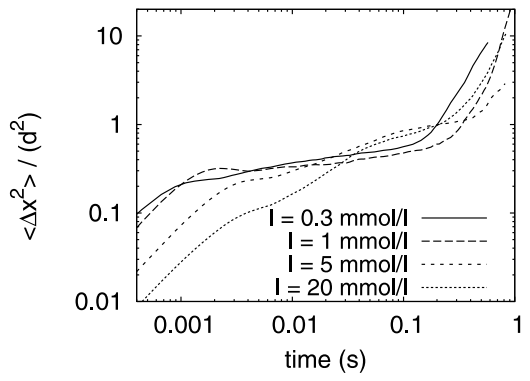

Fig. 6. Mean squared displacement at $p \mathrm{H}=6$ for different ionic strengths, without shear. One can see a ballistic regime for short times, a central plateau and a collective long time movement which can be a movement of a whole cluster or cage escape events of single particles. Depending on the ionic strength, the central plateau is more or less pronounced. A comparison of the plateau for different simulations can be used to decide, if a certain state belongs to the repulsive region of the stability diagram. A state well in the liquid microstructure should be used as a reference for the comparison (source: [19]) 
groups of particles move, or single particles can escape from a cage formed by its neighbors. Depending on the ionic strength different effects are important and thus the shape of the curve is different. For large ionic strengths the particles form clusters and these clusters may drift or rotate in the system. Then the collective motion is more dominant and the mean squared displacement grows faster than in single particle diffusion. The mean squared displacement does not show a plateau, then. But in the repulsive regime, the neighbors limit the motion of the particles, and the slope of the plateau is flatter, i.e., the plateau is even more pronounced, compared to the suspended case. In the repulsive regime the particles tend to arrange themselves in layers when shear is applied [17] and long range correlations can be found in un-sheared systems [20].

\subsection{Stability Diagram}

The results of the investigations presented up to here can be summarized in a stability diagram for our $\mathrm{Al}_{2} \mathrm{O}_{3}$-suspension (Fig. 7). Three different microstructures can be identified: a repulsive structure, a suspended region and a clustered region. In contrast to our previous work [17, 20], we have explored the parameter space more in the repulsive regime and deeper in the clustered region. We use the mean squared displacement, the correlation function, and the structure factor, to decide to which of the three microstructures a certain point in the stability diagram belongs. However, the borders between the re-

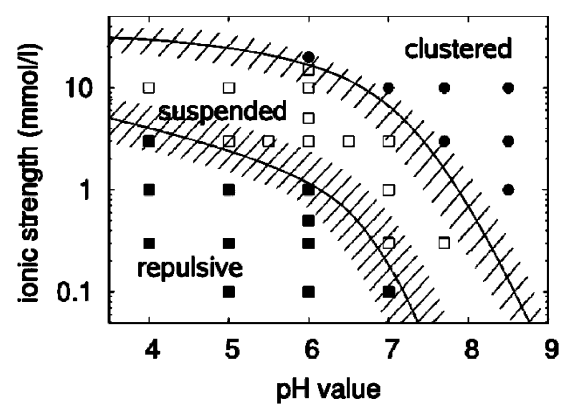

Fig. 7. Stability diagram (plotted for $\Phi=35 \%$ and without shear): depicting three regions: a clustered region (filled circles), a suspended regime (open squares), and a repulsive structure (filled squares). In the clustered region particles aggregate which leads to inhomogeneity in the system. In the suspended regime, the particles are distributed homogeneously in the system and they can move freely. In the repulsive regime the mobility of the particles is restricted by electrostatic repulsion exerted by their neighbors. As a result they arrange in a local order which maximizes nearest neighbor distances. The borders between the regimes are not sharp. They depend on the shear rate and on the volume fraction. Therefore we have indicated the crossover regions by the shaded patterns. The lines are guides to the eye (source: [19]) 
gions are not sharp and they depend on the shear rate. We have indicated the crossover regions by the shaded patterns in the stability diagram. If the volume fraction is decreased, the region of the repulsive structure becomes smaller.

To decide if a state is in the suspended region or in the repulsive one of the stability diagram, we have compared the plots of the mean squared displacement for the simulations without shear. If the plateau was pronounced there, we have counted the state among the repulsive regime. As a second criterion one can compare the pair correlation function. If there are long range correlations even though the system is not sheared, then the microstructure is the repulsive one. Finally, the shear force can be used to localize the border to the repulsive regime. For a given shear rate and a fixed volume fraction, the shear force depends on the particle interactions. If the shear force increases compared to a state well in the suspended regime, the motion of the particles is blocked by the electrostatic interaction in the repulsive regime.

Thus, to decide, if a state belongs to the clustered or to the suspended regime, we first study the snapshots of the system. If we see no clusters there, the clustered regime can be excluded. But, if we see clusters, we also consult numerical quantities like the density of clusters and the rate of cluster growth into account. Both, the density and the time for cluster growth are indications for the stability of the clusters. If they grow slowly and their density is low, we count the state to the suspended regime. The stability diagram obtained by these criteria is consistent with the results of the simulations with shear flow, shown in Fig. 2 and Fig. 5. Especially, the increased cluster formation for $I=3 \mathrm{mmol} / \mathrm{l}$ starting between $p \mathrm{H}=7-8$ is reflected in an increased nearest neighbor peak in Fig. 2, and low-k-peak in Fig. 5 respectively, and in a border between suspended and clustered regime in Fig. 7. The repulsive structure for $p \mathrm{H}=4$ and $I=3 \mathrm{mmol} / \mathrm{l}$ can not be recognized in Fig. 2 and Fig. 5 , but in a pronounced layer formation.

\section{Dilute Suspensions}

To study the process of cluster growth we simulate dilute suspensions of $\Phi=$ $5 \%$ only. To see cluster-cluster aggregation we scale up the simulation volume to $17.76 \mu \mathrm{m}^{3}$ containing $10560 \mathrm{MD}$ particles and $1.3 \cdot 10^{7}$ fluid particles. Due to the computational demands of the fluid solver, a single simulation of $5 \mathrm{~s}$ real time requires about $5000 \mathrm{CPU}$ hours on $32 \mathrm{CPUs}$ of a state of the art supercomputer.

We use a cluster counting algorithm [18] to detect clusters of particles in the suspension and to evaluate their size. In Fig. 8 we present the time dependence of the mean cluster size (a) and of the number of clusters in the system (b). We find that both observables can be fitted by a power law of the form $A \cdot(t+B)^{C}$, where $A, B, C$ are fitting parameters. The lines in the figure correspond to the fit and the symbols to the simulation data. It would 

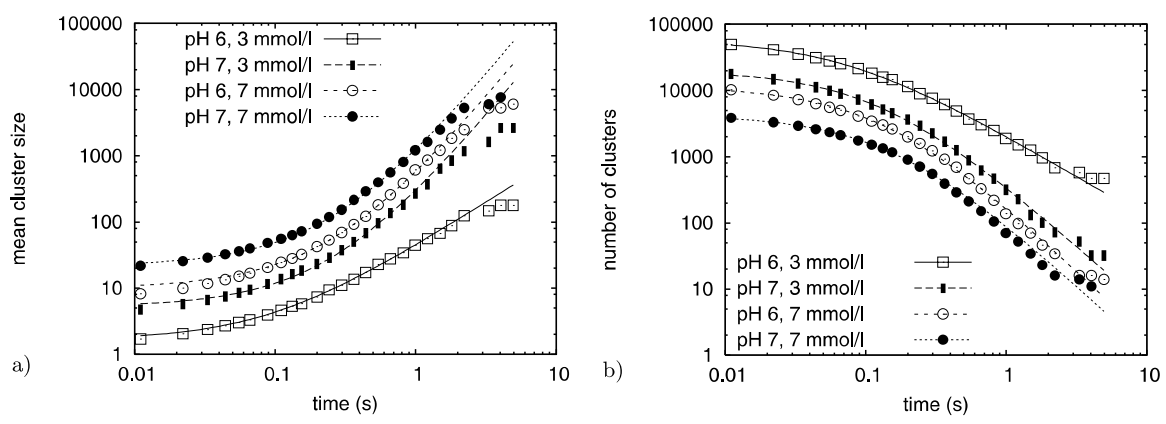

Fig. 8. The time dependence of the mean cluster size is plotted for different simulation parameters (a). Fig. b) depicts the time dependence of the number of clusters found in the system. Each curve is shifted vertically by a factor of two for better visibility. While the symbols correspond to simulation data, lines are given by a power law fit (source: [18])

be of great interest to investigate if a general scaling behavior can be observed depending on the volume concentration, the ionic strength and the $p \mathrm{H}$-value. However, for this a detailed investigation of the parameter space would be needed which will be the focus of a future work.

\section{Summary}

We have utilized our new parallel simulation code to model colloidal particles in shear flow and investigated how the clustering process due to attractive DLVO potentials is affected by the hydrodynamic forces. We find a consistent behavior on different length scales. The nearest neighbor peak of the pair correlation function has been used to observe the direct neighborhood of the particles and the low-k-peak of the structure factor to keep track of the length scales up to the system size. In both cases a suppression of the cluster formation by the shear flow can be seen at low $p \mathrm{H}$-values. For large $p \mathrm{H}$-values low shear rates even support the clustering process. In contrast, for high shear rates it suppresses the cluster formation. We have evaluated the mean squared displacement to characterize the repulsive regime. To summarize the results we have drawn the stability diagram as given in Fig. 7. To our knowledge this stability diagram for $\mathrm{Al}_{2} \mathrm{O}_{3}$ suspensions is reproduced quantitatively for the first time from simulations. It helps to predict the behavior of a real suspension. Our findings on the cluster formation process suggest that soft stirring can enhance the cluster formation in industrial processing of this material. Further investigations can be carried out on the fractal dimension and its dependence on the experimental conditions. The low-k-peak of the structure factor can be used for that. We have followed up this point by simulating dilute suspensions for selected conditions. We have found that 
the time dependence of the mean cluster size and the number of clusters in the system can be well described by power laws.

\section{Acknowledgements}

We thank H.J. Herrmann for valuable collaboration and his support. The High Performance Computing Center Stuttgart, the Scientific Supercomputing Center Karlsruhe and the Neumann Institute for Computing in Jülich are highly acknowledged for providing the computing time and the technical support needed for our research. This work was partly financed by the German Research Foundation (DFG) within the project DFG-FOR 371 "Peloide".

\section{References}

1. B. J. Ackerson, C. G. DeKruif, N. J. Wagner, and W. B. Russel. Comparison of small shear flow rate-small wave vector static structure factor data with theory. J. Chem. Phys., 90(6):3250, 1989.

2. B. J. Ackerson and P. N. Pusey. Shear-induced order in suspensions of hard spheres. Phys. Rev. Lett., 61(8):1033, 1988.

3. S. Alexander, P. M. Chaikin, P. Grant, G. J. Morales, P. Pincus, and D. Hone. Charge renormalization, osmotic pressure, and bulk modulus of colloidal crystals: Theory. J. Chem. Phys., 80(11):5776-81, 1984.

4. I. Ali, D. Marenduzzo, and J. M. Yeomans. Dynamics of polymer packaging. J. Chem. Phys., 121:8635-8641, Nov. 2004.

5. M. P. Allen and D. J. Tildesley. Computer simulation of liquids. Oxford Science Publications. Clarendon Press, Oxford, 1987.

6. J. F. Brady. The rheological behavior of concentrated colloidal suspensions. J. Chem. Phys., 99(1):567-81, 1993.

7. J. F. Brady and G. Bossis. Stokesian dynamics. Ann. Rev. Fluid Mech., 20:111$57,1988$.

8. A. A. Catherall, J. R. Melrose, and R. C. Ball. Shear thickening and orderdisorder effects in concentrated colloids at high shear rates. Journal of Rheology, 44(1):1-25, 2000.

9. I. Cohen, T. G. Mason, and D. A. Weitz. Shear-induced configurations of confined colloidal suspensions. Phys. Rev. Lett., 93(4):046001, 2004.

10. A. de Candia, E. del Gado, A. Fierro, N. Sator, and A. Coniglio. Colloidal gelation, percolation and structural arrest. Physica A, 358:239-248, 2005.

11. B. V. Derjaguin and L. D. Landau. Theory of the stability of strongly charged lyophobic sols and of the adhesion of strongly charged particles in solutions of electrolytes. Acta Phsicochimica USSR, 14:633, 1941.

12. J. Dobnikar, Y. Chen, R. Rzehak, and H. H. von Grünberg. Many-body interactions in colloidal suspensions. J. Phys.: Condens. Matter, 15:S263, 2003.

13. J. Dobnikar, R. Rzehak, and H. H. von Grünberg. Effect of many-body interactions on the solid-liquid phase behavior of charge-stabilized colloidal suspensions. Europhys. Lett., 61(5):695-701, 2003. 
14. E. Falck, J. M. Lahtinen, I. Vattulainen, and T. Ala-Nissila. Influence of hydrodynamics on many-particle diffusion in 2 d colloidal suspensions. Eur. Phys. J. E, 13:267-275, 2004.

15. M. J. Grimson and M. Silbert. A self-consistent theory of the effective interactions in charge-stabilized colloidal dispersions. Macromol. Phys., 74(2):397-404, 1991.

16. L. Harnau and S. Dietrich. Depletion potential in colloidal mixtures of hard spheres and paltelets. Phys. Rev. E, 69:051501, 2004.

17. M. Hecht, J. Harting, M. Bier, J. Reinshagen, and H. J. Herrmann. Shear viscosity of clay-like colloids in computer simulations and experiments. Phys. Rev. E, 74:021403, 2006.

18. M. Hecht, J. Harting, and H. J. Herrmann. Formation and growth of clusters in colloidal suspensions. Int. J. Mod. Phys. C, 2007. in print.

19. M. Hecht, J. Harting, and H. J. Herrmann. A stability diagram for dense suspensions of model colloidal al2o3-particles in shear flow. arXiv:cond-mat/0606455, 2006. Accepted for publication in Phys. Rev. E.

20. M. Hecht, J. Harting, T. Ihle, and H. J. Herrmann. Simulation of claylike colloids. Phys. Rev. E, 72:011408, jul 2005.

21. M. Hütter. Brownian Dynamics Simulation of Stable and of Coagulating Colloids in Aqueous Suspension. PhD thesis, Swiss Federal Institute of Technology Zurich, 1999.

22. M. Hütter. Local structure evolution in particle network formation studied by brownian dynamics simulation. J. Colloid Interface Sci., 231:337-350, 2000.

23. A.-P. Hynninen, M. Dijkstra, and R. van Roij. Effect of three-body interactions on the phase behavior of charge-stabilized colloidal suspensions. Phys. Rev. E, 69:061407, 2004.

24. T. Ihle and D. M. Kroll. Stochastic rotation dynamics I: Formalism, galilean invariance, green-kubo relations. Phys. Rev. E, 67(6):066705, 2003.

25. T. Ihle and D. M. Kroll. Stochastic rotation dynamics II: Transport coefficients, numerics, long time tails. Phys. Rev. E, 67(6):066706, 2003.

26. Y. Inoue, Y. Chen, and H. Ohashi. Development of a simulation model for solid objects suspended in a fluctuating fluid. J. Stat. Phys., 107(1):85-100, 2002.

27. A. Komnik, J. Harting, and H. J. Herrmann. Transport phenomena and structuring in shear flow of suspensions near solid walls. Journal of Statistical Mechanics: theory and experiment, P12003, 2004.

28. A. J. C. Ladd. Numerical simulations of particulate suspensions via a discretized boltzmann equation. part 1. theoretical foundation. J. Fluid Mech., 271:285-309, 1994.

29. A. J. C. Ladd. Numerical simulations of particulate suspensions via a discretized boltzmann equation. part 2. numerical results. J. Fluid Mech., 271:311-339, 1994.

30. A. J. C. Ladd and R. Verberg. Lattice-boltzmann simulations of particle-fluid suspensions. J. Stat. Phys., 104(5):1191, 2001.

31. M. Lattuada, H. Wu, and M. Morbidelli. Estimation of fractal dimension of colloidal gels in the presence of multiple scattering. Phys. Rev. E, 64:061404, 2001.

32. Y. Levin, T. Trizac, and L. Bocquet. On the fluid-fluid phase separation in charged-stabilized colloidal suspensions. J. Phys.: Condens. Matter, 15:S3523, 2003 . 
33. A. Malevanets and R. Kapral. Mesoscopic model for solvent dynamics. J. Chem. Phys., 110:8605, 1999.

34. A. Malevanets and R. Kapral. Solute molecular dynamics in a mesoscale solvent. J. Chem. Phys., 112:7260, 2000.

35. D. W. McCarthy, J. E. Mark, and D. W. Schaefer. Synnthesis, structure, and properdies of hybrid organic-inorganic composites based on polysiloxanes. i. poly(dimathylsiloxane) elastomers containing silica. J. Polym. Sci. B, 36(7):1167, 1998.

36. J. R. Melrose and D. M. Heyes. Simulations of electrorheological and particle mixture suspensions: Agglomerate and layer structures. J. Chem. Phys., 98(7):5873-5886, 1993.

37. R. Oberacker, J. Reinshagen, H. von Both, and M. J. Hoffmann. Ceramic slurries with bimodal particle size distributions: Rheology, suspension structure and behaviour during pressure filtration. In N. C. S. Hirano, G.L. Messing, editor, Ceramic Processing Science VI, volume 112, pages 179-184. American Ceramic Society, Westerville, OH (USA), 2001. ISBN 1574981048.

38. J. T. Padding and A. A. Louis. Hydrodynamic and brownian fluctuations in sedimenting suspensions. Phys. Rev. Lett., 93:220601, 2004.

39. J. T. Padding and A. A. Louis. Hydrodynamic interactions and brownian forces in colloidal suspensions: Coarse-graining over time and length-scales. Phys. Rev. E, 74:031402, 2006.

40. T. Palberg, W. Mönch, F. Bitzer, R. Piazza, and T. Bellini. Freezing transition for colloids with adjustable charge: A test of charge renormalization. Phys. Rev. Lett., 74:4555, 1995.

41. T. N. Phung, J. F. Brady, and G. Bossis. Stokesian dynamics simulation of brownian suspensions. J. Fluid Mech., 313:181-207, 1996.

42. M. Ripoll, K. Mussawisade, R. G. Winkler, and G. Gompper. Low-reynoldsnumber hydrodynamics of complex fluids by multi-particle-collision dynamics. Europhys. Lett., 68:106-12, 2004.

43. M. Ripoll, K. Mussawisade, R. G. Winkler, and G. Gompper. Dynamic regimes of fluids simulated by multiparticle-collision dynamics. Phys. Rev. E, 72:016701, 2005.

44. M. Ripoll, R. G. Winkler, and G. Gompper. Star polymers in shear flow. Phys. Rev. Lett., 96:188302, 2006.

45. A. Sierou and J. F. Brady. Accelerated stokesian dynamics simulations. J. Fluid Mech., 448:115, 2001.

46. A. Sierou and J. F. Brady. Shear-induced self-diffusion in non-colloidal suspensions. J. Fluid Mech., 506:285, 2004.

47. L. E. Silbert, J. R. Melrose, and R. C. Ball. Colloidal microdynamics: Pairdrag simulations of model-concentrated aggregated systems. Phys. Rev. E, 56(6):7067-7077, December 1997.

48. K. G. Soga, J. R. Melrose, and R. C. Ball. Continuum percolation and depletion flocculation. J. Chem. Phys., 108(14):6026-6032, 1998.

49. K. G. Soga, J. R. Melrose, and R. C. Ball. Metastable states and the kinetics of colloid phase separation. J. Chem. Phys., 110(4):2280-2288, 1999.

50. V. Trappe, V. Prasad, L. Cipelletti, P. N. Segre, and D. A. Weitz. Jamming phase diagram for attractive particles. Nature, 411(3):772-774, 2001.

51. R. van Roij and J.-P. Hansen. Van der waals-like instability in suspensions of mutually repelling charged colloids. Phys. Rev. Lett., 79(16):3082-85, 1997. 
52. E. J. W. Vervey and J. T. G. Overbeek. Theory of the Stability of Lyophobic Colloids. Elsevier, Amsterdam, 1948.

53. R. G. Winkler, K. Mussawisade, M. Ripoll, and G. Gompper. Rod-like colloids and polymers in shear flow: a multi-particle-collision dynamics study. J. Phys.: Condens. Matter, 16(38):S3941-54, 2004.

54. R. Yamamoto, K. Kim, Y. Nakayama, K. Miyazaki, and D. R. Reichman. On the role of hydrodynamic interactions in colloidal gelation. arXiv:condmat/0604404, 2006. 